\title{
Hyperthelie, Wurfgröße und Trächtigkeitsdauer bei der Unterfamilie Tenrecinae Cabrera, 1925 (Mammalia: Insectivora: Tenrecidae), nebst Bemerkungen zur Längsstreifung der Gattung Hemicentetes
}

\author{
Walter Poduschka \\ Zoologisches Institut der Universität Wien, Althanstr. 9, A-1090 Wien; Insectivore Research Center, \\ Wultendorf 98, A-2134 Staatz, Österreich
}

Keywords: Insectivora, Tenrecidae, hyperthelia, reproduction, litter size, pregnancy, mimicry

\begin{abstract}
In a comparison of basic reproductive variables (number of teats, litter size, and duration of pregnancy) in the Madagascan Tenrecinae, some striking features (hyperthelia, litter size) are observed in Tenrec ecaudatus Lacépède, 1799 and the genus Hemicentetes Mivart, 1871. These variations are compared to Setifer setosus Froriep, 1806 and Echinops telfairi Martin, 1838. Tenrec ecaudatus gives birth to litters at intervals shorter than what was once believed to be the duration of pregnancy. This could be due either to superfetation, or to giving birth to one litter in two or more installments.

The longitudinal stripe pattern of the genus Hemicentetes is interpreted as a mimicry of the sympatric, equally striped, juvenile Tenrec ecaudatus. The latter species protects its young with attending defensive-aggressive parents. The reproductive strategies of the Tenrecinae are discussed.
\end{abstract}

\section{Zusammenfassung}

Bei einer vergleichenden Untersuchung unterschiedlicher Fortpflanzungseigenheiten (Zitzenzahl, Wurfgröße, Trächtigkeitsdauer) der madagassischen Unterfamilie Tenrecinae wurden einige frappante Unterschiede zwischen Tenrec ecaudatus Lacépède, 1799 und der Gattung Hemicentetes Mivart, 1871 einerseits und Setifer setosus Froriep, 1806 und Echinops telfairi Martin, 1838 andererseits gefunden. Besonders bemerkenswert sind Austreibungen bei Tenrec ecaudatus in Intervallen, die wesentlich kürzer sind als die normale Tragzeit. Superfetation oder andere Erklärungen wären denkbar; faktisch kann der Gesamtwurf in zwei oder mehr Raten zur Welt kommen.

Die Längsstreifung von Hemicentetes wird versuchsweise gedeutet als Mimikry der Jungtiere mit den sympatrisch lebenden (ebenfalls gestreiften) jungen Tenrec ecaudatus, die in diesem Alter von ihren wehrhaften Elternteilen erfolgreich verteidigt werden. Die Reproduktionsstrategie der Tenrecinen wird diskutiert.

\section{Einleitung}

Die Unterfamilie Tenrecinae Cabrera, 1925 besteht aus 4 Gattungen mit insgesamt 5 Arten:

Tenrec ecaudatus Lacépède, 1799

Hemicentetes Mivart, 1871

H. semispinosus G. Cuvier, 1798

H. nigriceps Günther, 1875

Setifer setosus Froriep, 1806

Echinops telfairi Martin, 1838

Die früheste Literatur beschränkte sich auf Erstbeschreibungen und taxonomische Meinungsverschiedenheiten (Buffon, 1764; Lacépède, 1799; I. Geoffroy Saint-Hilaire, 1839; Jentinck, 1879; etc.). Erst später befaßte man sich mit morphologischen Themen, wie etwa Osteologie, Odontologie, Anatomie und Myologie (Mivart, 1871; Dobson, 1882; Leche, 1907), seltener mit anderen somatischen Einzelheiten. Speziell zum bemerkenswert heterogenen Fortpflanzungskomplex liegen indessen ausführliche Arbeiten über die Frühentwicklung der Unterfamilie Tenrecinae vor. Wir verdanken vor allem Bluntschli $(1932,1937,1938 \mathrm{a}, \mathrm{b})$ und seinem Kreis (R.H. Goetz, F. Strauss, C. Leimgruber, und K. Feremutsch) diesbezüglich erhebliches Wissen.

An der Erforschung lebender Tenrecinen und an Ergänzungen ihrer Erstbeschreibungen war man bis weit in unser Jahrhundert wenig interessiert. Dies änderte sich erst mit den breitgefächerten Themenspektren neuerer Autoren.

Es fällt aber auf, daß die Korrelierung fortpflanzungsbezogener, jedoch verschiedenen Fragenkreisen angehöriger Fakten nicht versucht 


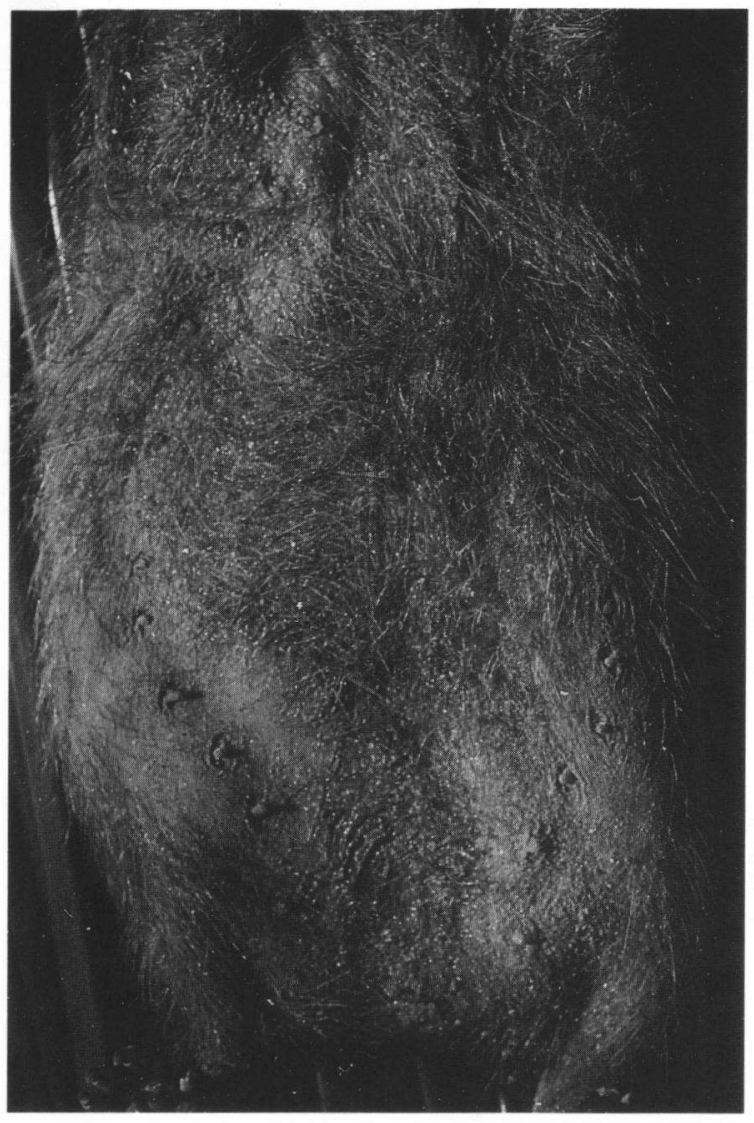

Abb. 1. Tenrec ecaudatus, \%, Ventralseite. Beachte die in regelmäßigen Abständen von inguinal bis axillar verlaufende Zitzenreihe. (Photographie W. Poduschka.)

wurde. Dazu gehören die innerhalb der Unterfamilie Tenrecinae keineswegs konformen Beziehungen zwischen Zitzen- und Embryonenzahlen, Wurfgröße, Trächtigkeitsdauer und den in manchen Fällen erstaunlichen Besonderheiten des Geburtsverlaufes. All dies ist das Thema dieser Arbeit.

\section{Hyperthelie}

Nach Romer \& Parsons (1983) steht die Zahl der als modifizierte apokrine Drüsen angesehenen Mamillen im allgemeinen in Beziehung zur Zahl der Jungen, die üblicherweise geboren werden. Die meisten multiparen Säugetiere haben 4-6 Milchzitzen, doch sind sie bei vielen Metatheria und auch Eutheria - etwa der Vielzitzenmaus (Gattung Mastomys Thomas, 1882) - beträchtlich zahlreicher. Eine Übersicht findet sich bei Starck (1982). Zumeist sind die Zitzen im Verlauf der embryonal angelegten Milchleiste, nicht selten jedoch in einer thorakalen und/oder einer abdominalen Gruppe angeordnet.

Breslau (1910) wies darauf hin, daß überzählige Milchdrüsen embryonal häufiger und als Reste einer ursprünglich größeren, im Ablauf der Phylogenie der Säugetiere aber reduzierten Milchdrüsenzahl anzusehen seien, nicht jedoch mit der Steigerung der Fruchtbarkeit korreliert wären.

Zitzenüberzahl ist auch bei Insectivora bekannt, doch gibt es Unklarheiten: Weber (1927) gab im 1. Band seines grundlegenden Werkes als maximale Zitzenanzahl bei den Monodelphia für "Centetes" (= Tenrec ecaudatus) 22 (S. 43), im 2. Band (S. 95) nur 12 abdominalo-inguinale an. Vermutlich irrtümlich schloß er hier auch "Ericulus" (= Setifer) ein, bei welchem die Zahl 12 korrekt ist. Da er jedoch im späteren Verlauf dieses Satzes von Zitzenpaaren schreibt, sind hier wohl Flüchtigkeitsfehler anzunehmen. Auch bei Starck (1982) findet sich in einer Liste der Zitzenzahlen verschiedener Säuger ein offensichtlicher Druckfehler: Für Tenrec werden 19-25 Paare - somit 38-50 Zitzen - angegeben. Nur im Falle einer (in der Arbeit nicht erwähnten) Verdoppelung der Milchleiste hätte die zweitgenannte Anzahl ventral Platz, andernfalls ist sie topographisch unmöglich. Die korrekte Zitzenzahl lautet 12-14 Paare; der Zwischenraum zwischen den Zitzen beträgt bei ausgewachsenen Exemplaren maximal $20 \mathrm{~mm}$ (Abb. 1). Auf das Vorkommen doppelter Milchleisten bei Säugetieren wies schon Breslau (1910) hin; Starck (1982) führte dies weiter. Bauer (1960) fand eine doppelt angelegte Milchleiste bei einem Exemplar der Spitzmaus Neomys anomalus Cabrera, 1907, hielt dies aber für einen Sonderfall.

Hyperthelie bei der eurasiatischen Igelgattung Erinaceus wurde von Firbas \& Poduschka (1971) beschrieben, scheint aber selten zu sein (von 475 untersuchten Exemplaren zeigten 4 Hyperthelie = $0,85 \%$ ). Gleichwohl besteht bei Erinaceinen keine Veranlassung für eine Überzahl, da die Wurfstärke nur selten die Zitzenzahl übersteigt (Poduschka, unveröff.). 
Tab. I. Bisherige Angaben zur Zitzen- und Embryonenzahl von T. ecaudatus.

\begin{tabular}{cll}
\hline Zitzenzahl & Embryonen & Referent \\
\hline- & 32 & Bluntschli (1932) \\
- & 25 & Rand (1935) \\
$24-28$ & $(32), 16,14$ & Goetz (1936) \\
$12-20$ & - & Leimgruber (1939) \\
12 & - & Petter \& Petter- \\
& & Rousseaux (1963) \\
$22-27$ & 16 & Racey \& Nicoll (1984) \\
$24-28$ & - & Poduschka (s.o. Abb. 1) \\
\hline
\end{tabular}

'Goetz referierte über dasselbe Exemplar wie Bluntschli (1932).

Bei den Tenrecinen-Gattungen Tenrec und $\mathrm{He}$ micentetes hingegen sind sowohl Hyperthelie als auch ungewöhnliche Wurfgrößen häufig (Tab. I). Dies ist bei den restlichen Gattungen der Unterfamilie, Setifer und Echinops, nicht der Fall.

\section{Tenrec ecaudatus}

$\mathrm{Da}$ diese Gattung unter den Säugetieren die zahlenmäßig stärksten Würfe erbringt, war schon frühen Autoren bekannt. Bluntschli (1932) gibt als "gewöhnliche" Embryonenzahl 24 an, zitiert aber auch die Höchstzahl von 32. Über die größten, bisher bekannten Lebendwürfe wird im zweiten Teil dieser Arbeit referiert. In Tab. I werden die bisher gemeldeten Zitzenzahlen den Embryonenzahlen gegenübergestellt.

\section{Hemicentetes}

Zitzenzahl. - Darüber gab es bisher nur zwei Meldungen (Grandidier \& Petit, 1930; Bluntschli, 1937), wonach 18 bzw. 20 Zitzen vorkämen. Wir können dies bestätigen. An zwei Sammlungsexemplaren (BMNH Nr. 35.1.8.192 und 35.1.8. 202) des British Museum (Natural History) meinen wir, je eine - allerdings nur partielle - doppelte Zitzenreihe, median der normalen, entdeckt $\mathrm{zu}$ haben, doch ist der Erhaltungszustand der betreffenden Bälge schlecht.

In den Sammlungen der Académie Malgache und des Muséum National d'Histoire Naturelle, Paris (MNHN) fanden wir mehrere Bälge von ○ , die entweder während ihrer Säugeperiode getötet worden waren oder schon mehrfach geboren hatten; ihre Zitzen waren stark ausgeprägt und dadurch gut sichtbar. Bei einem Balg (MNHN Nr. 1932/3443) sind sie durch die enthaarten Zitzenhöfe besonders deutlich (Abb. 2). Ob diese Haare zu Beginn der Laktationsperiode von selbst ausfallen oder von der Mutter entfernt werden, damit die langgezogenen, zarten Schnauzen der Neonaten die kaum $2 \mathrm{~mm}$ langen Zitzen besser fassen können, ist unklar.

Embryonenzahl. - Bauchot \& Stephan (1964) untersuchten 4 \& $\%$, von denen zwei je 9 Embryonen enthielten.

\section{Setifer setosus}

Diese Gattung wurde von mehreren Autoren bearbeitet und auch gezüchtet (Strauss, 1939a,b, 1942, 1943, 1944, 1946; Eisenberg \& Gould, 1967; Eisenberg \& Muckenhirn, 1968; Riordan, 1972; Mallinson, 1972; Poduschka, 1974). Keiner hat an dieser Gattung jemals Hyperthelie festgestellt; normal sind 6 Zitzenpaare.

\section{Echinops telfairi}

Auch diese Gattung wurde von mehreren Autoren gehalten bzw. gezüchtet: Eibl-Eibesfeldt (1965), Honegger \& Noth (1966), Eisenberg \& Gould (1967), Eisenberg \& Muckenhirn (1968), Mallinson (1968), und Riordan (1972). Ich selbst züchte sie seit 1971. Berichte über Hyperthelie bei Echinops sind mir unbekannt. Die Zitzenzahl beträgt 6 Paare.

\section{Wurfgröße}

Um Fehlerquellen auszuschalten, können zur Feststellung der Höchstzahl von Neonaten ausnahmslos nur Würfe aus Gefangenschaftshaltungen herangezogen werden. Aufgrund der seltenen Zuchterfolge bei der auch in dieser Hinsicht sehr heterogenen Unterfamilie ist die Ermittlung einer durchschnittlichen Jungenanzahl nicht aussagekräftig, da sowohl kopfstarke, als auch kleine Würfe vorkommen (siehe unten). 


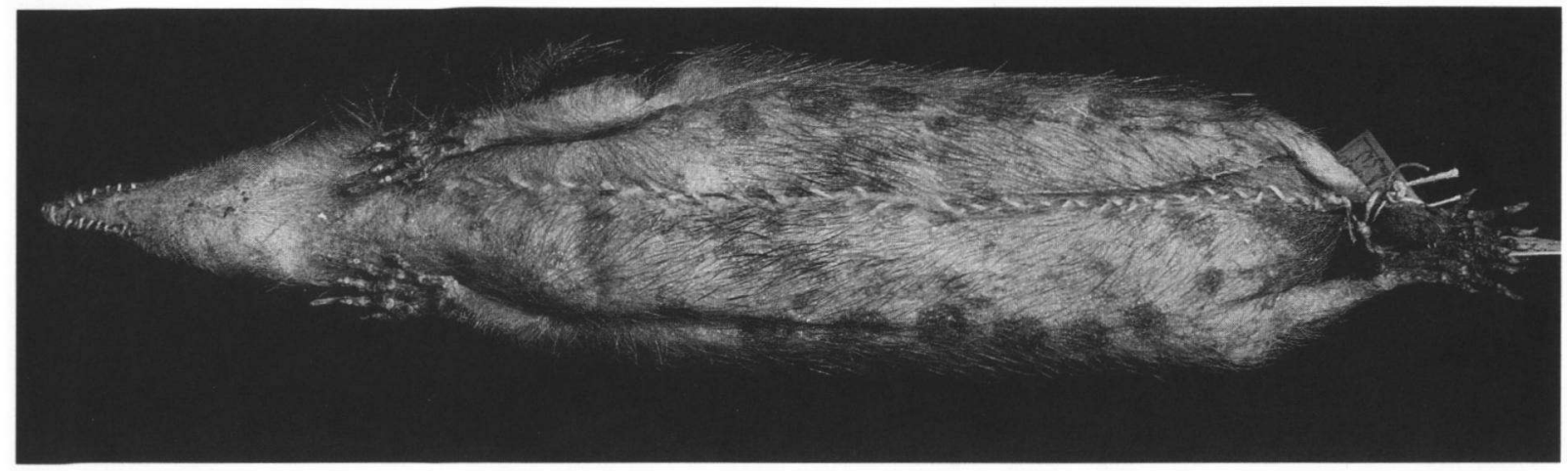

Abb. 2. Hemicentetes semispinosus, १, Zitzenreihe, erkennbar durch die dunkle Aureole. Die Zitzen selbst vertrocknen bei den meisten Präparaten aufgrund ihres geringen Durchmessers (weniger als $1 \mathrm{~mm}$ ) und brechen ab. Auf diesem Bild ist nur die letzte (?) Inguinalzitze rechts noch erhalten. (Photographie W. Poduschka.)

\section{Tenrec ecaudatus}

Details der von $T$. ecaudatus bekannten Würfe sind angegeben in Tab. II.

\section{Hemicentetes}

Bluntschli (1937) gibt die Jungenanzahl mit 6-10 an. Francke (1961) berichtet von einem Wurf (2 Jungtiere) importierter H. semispinosus im Zoo von Berlin. Gould \& Eisenberg (1966) hatten 1963 in Madagascar 4 Würfe von $H$. semispinosus gefunden, weitere 5 fielen im Laboratorium. $\mathrm{Ob}$ sie dort auch gezeugt wurden, wird nicht berichtet. Der stärkste Wurf hatte 11 Neonati. Ich selbst hatte 3 Würfe von $H$. semispinosus in Madagascar und insgesamt 8 von $H$. nigriceps in Wien, von welchen die beiden stärksten je 8 Jungtiere erbrachten.

\section{Setifer}

Mallinson (1972) und Riordan (1972) berichten von 13 Würfen im Zoo von Jersey; 2 Würfe hatten (als Höchstzahl) je 7 Jungtiere. In meiner Zucht fielen zwischen 1970 und September 1995 aus 16 Würfen insgesamt 46 Jungtiere, die maximale Jungenzahl war 6.

\section{Echinops}

Gould \& Eisenberg (1966) melden von 12 ㅇ dreizehn Würfe mit insgesamt 76 Jungtieren. Die
Wurfgrößen waren $1 \times 2,1 \times 3,1 \times 4,2 \times 5,3 \times 6,3 \times 7$, $1 \times 8,1 \times 10$. Riordan (1972) zählte im Jersey Zoo 9 Würfe; die Höchstzahl der Neonati war 5. Ich selbst hatte zwischen 1971 und 1994 aus 14 Würfen 38 Jungtiere $(4 \times 1,3 \times 2,3 \times 3,2 \times 4,1 \times 5$, $1 \times 6)$.

\section{Tragzeit}

Während die Tragzeit der hartstacheligen Tenrecinen Setifer und Echinops bekannt ist (vergl. Tab. III), gibt es diesbezüglich von Hemicentetes nur Annäherungswerte, wie die von Francke (1961) "mehr als 50 Tage", Gould \& Eisenberg (1966) "maximal 55 Tage" und Eisenberg \& Muckenhirn (1968) "mehr als 45, weniger als 55 Tage".

Der bei Tenrec ecaudatus festgestellte Sonderfall wird zuletzt bearbeitet.

\section{Tenrec ecaudatus}

Präzise Angaben liegen von Louwman (1973a, b) vor, der am 1. März 1972 einige Paarungen beobachten konnte. Am 22. April wurden 31 Jungtieren geboren. Die Tragzeit beträgt also mindestens 53 Tage. Die Richtigkeit der von Eisenberg \& Muckenhirn (1968) angegebenen 58-64 Tage hängt wohl davon $\mathrm{ab}$, ob schon vor der Mindestanzahl an Tagen (nicht registrierte) Kopulationen stattgefunden hatten, wie von Eisenberg (in litt.) eingeräumt wurde. 
Tab. II. Details der von T. ecaudatus bekannten Würfe.

\begin{tabular}{|c|c|c|c|}
\hline Wurfdatum & Neonatenzahl & Referent & Staat \\
\hline 30 Juni '31 & 21 & Goetz & Schweiz \\
\hline$?$ & $1-4$ & Eisenberg \& Muckenhirn (1968) & USA \\
\hline 11 Feb. '71 & 19 & Louwman (1973a) & Holland \\
\hline März '72 & 1 od. $2^{\prime}$ & Louwman (1973a) & Holland \\
\hline März '72 & 1 od. $2^{\prime}$ & Louwman (1973a) & Holland \\
\hline März '72 & 1 od. $2^{\prime}$ & Louwman (1973a) & Holland \\
\hline 22 Apr. '72 & 31 & Louwman (1973a) & Holland \\
\hline$?$ & 21 & Starck (1974) & Deutschland \\
\hline 19 Dez. '78 & $8^{2}$ & Andriatsarafara (1981) & Madagascar \\
\hline 3 Jan. '79 & $19^{2}$ & Andriatsarafara (1981) & Madagascar \\
\hline 27 Dez. '79 & 10 & Andriatsarafara (1981) & Madagascar \\
\hline 2 Dez. '85 & $2^{3}$ & Poduschka, diese Arbeit & Madagascar \\
\hline 11 Dez. '85 & $2^{3}$ & Poduschka, diese Arbeit & Österreich \\
\hline 22 Dez. '85 & $10^{3}$ & Poduschka, diese Arbeit & Österreich \\
\hline 30 Sept. '86 & $28+1$ ex & Neugebauer (pers. Mitt.) & Deutschland \\
\hline
\end{tabular}

'Die drei holländischen Würfe von März 1972 kamen von drei $\$$ Q, die ihrerseits erst 11 Monate vorher in Wassenaar/Holland geboren worden waren. Ihre (wenigen) Jungtiere starben jedoch alle am nächsten Tag (Louwman, 1973a). Möglicherweise zeigt dies an, daß primipare Tenrec $\&$ \& eher kleine Würfe haben und/oder stressempfindlich sind.

${ }^{2}$ Die beiden Würfe kamen von einem Weibchen im Abstand von nur 15 Tagen.

${ }^{3}$ Diese drei (!), kurz aufeinander folgenden Würfe kamen von nur zwei (!) Muttertieren.

Tab. III. Tragezeit von Setifer und Echinops.

\begin{tabular}{lll}
\hline Species & Tragezeit & Referent \\
\hline S. setosus & $62-69^{* *}$ & Eisenberg \& Gould (1967) \\
& $65-69^{* *}$ & Eisenberg \& Muckenhirn (1968) \\
& $63^{* *}$ & Eisenberg \& Gould (1970) \\
& $51-56$ & Riordan (1972) \\
& 54 & Mallinson (1972) \\
& 54 & Poduschka (1974) \\
E. telfairi & $62-65$ & Eisenberg \& Muckenhim (1968) \\
& 64 & Mallinson (1968) \\
& 62 & Poduschka (unveröff.) \\
\hline
\end{tabular}

"Nach Eisenberg (briefl. Mitt.) können die Paarungen bereits einige Tage früher begonnen haben, was die lange, aber unsichere Zeitspanne erklärt.

Die genannten Tragzeiten entsprechen wenigstens ungefähr jenen von Setifer und Echinops. Bei $T$. ecaudatus kommt aber eine bis jetzt unerklärte Sondermodalität vor, aus deren Gegenüberstellung mit den fortpflanzungsbiologischen Details bei den Gattungen Setifer und Echinops sich interessante Aspekte ergeben. Sie konnte bis jetzt von zwei Autoren beobachtet werden, was indessen noch keinen Schluß auf ihre Häufigkeit zuläßt.
(1) Im Zuge seiner Diplomarbeit über die Ontogenie und Ethologie von T. ecaudatus bekam Andriatsarafara (1981) am 11. Dezember 1978 ein adultes Pärchen aus Baroboka. Das $\$$ gebar am 19. Dezember 1978 acht Jungtiere, die aber am nächsten Tag von den Elterntieren in der Erde des Terrariums vergraben wurden und starben. Die Ursache dieses Fehlverhaltens ist wohl darin $\mathbf{z u}$ sehen, daß die Tiere - zur vermeintlich besseren Beobachtungsmöglichkeit - ohne jedwede Dekkung gehalten wurden. Daher wurden ab diesem Geschehen Grünpflanzenteile zum Bau eines Nestes zur Verfügung gestellt. Nach nur 25 Tagen, am 13. Januar 1979, brachte das \& 19 weitere gesunde Jungtiere zur Welt. Ob die schnelle Aufeinanderfolgung der Wurfteile auf Superfetation zurückgeführt werden kann, ist unbekannt. Wie die Berichte von Eisenberg \& Muckenhirn (1968) und Louwman $(1973 a, b)$ zeigen, ist sie jedenfalls nicht die Regel.

(2) Am Ende einer längeren Feldarbeit südöstlich von Ranarantsoa (Madagascar) erhielten wir am 1. Dezember 1985 von Dorfbewohnern u.a. auch 3 


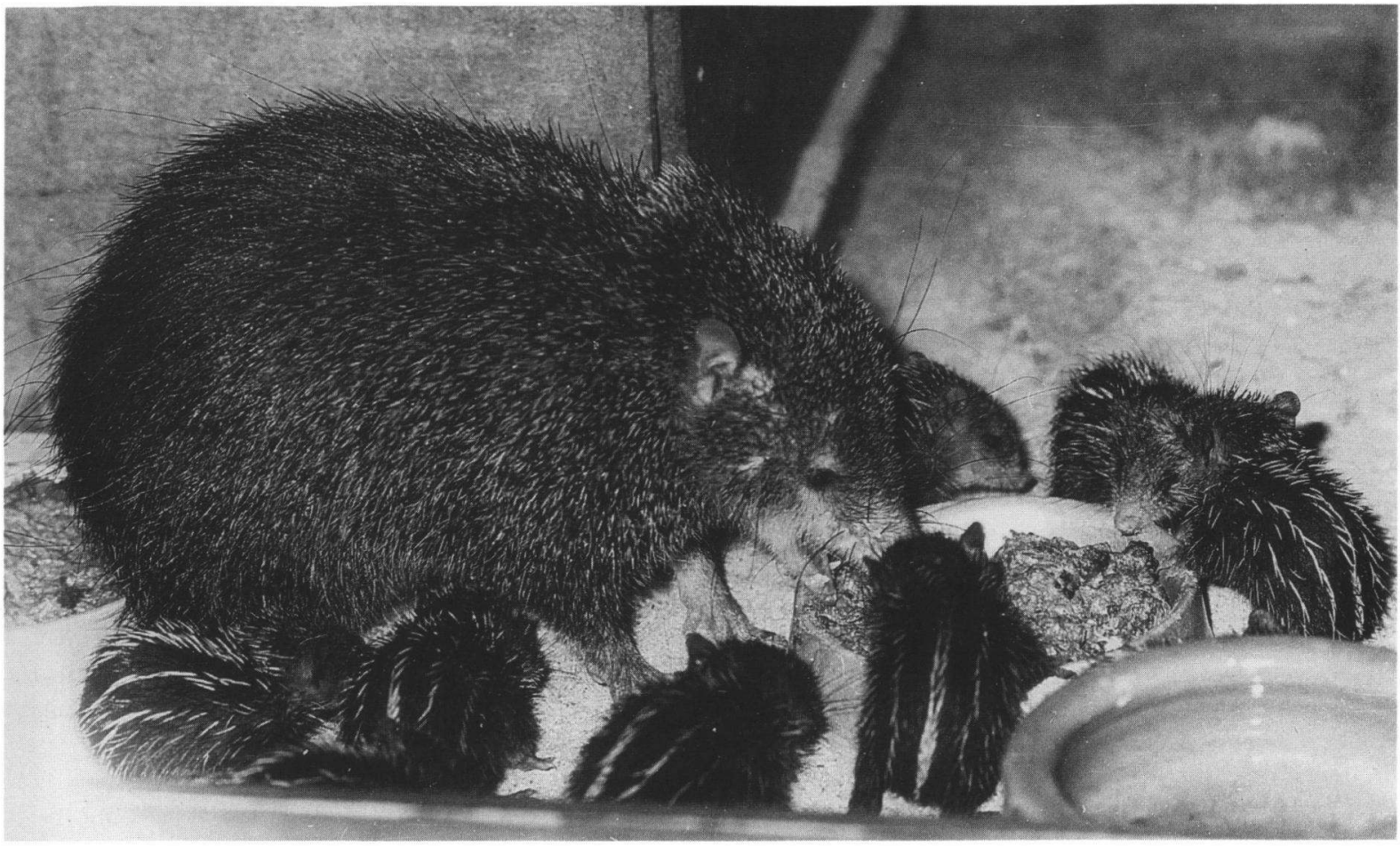

Abb. 3. Tenrec ecaudatus, Muttertier mit 9 (sichtbaren) Jungtieren. Beachte die schütteren Längsstreifen der etwa 10 Tage alten Jungtiere und das bei den beiden Exemplaren rechts vorne gut sichtbare Stridulationsorgan im Medianstreifen am Hinterrücken. (Photographie W. Poduschka.)

adulte $T$. ecaudatus ( 1 o $^{\prime}$ und 2 \% $ᄋ$ ). Die Tiere erschienen wohlgenährt, doch zeigten die beiden 우 keine Anzeichen von Trächtigkeit und wurden daher während der strapaziösen Nachtfahrt zur Ausgangsstation über holperige, überschwemmte Straßen nicht voneinander getrennt. Da unsere Heimreise kurz bevorstand, deponierten wir sie am frühen Morgen in einem kleinen Käfig im zoologischen Garten Tsimbazaza in Tananarivo. Möglicherweise hatten aber die 14 Stunden andauernden Geholpers den Beginn einer (frühzeitigen ?) Geburt eingeleitet, denn am nächsten Morgen (2. Dezember 1985) waren im Käfig der drei Tenrec-Exemplare auch zwei anscheinend gesunde Neonaten, deren Entwicklungszustand nicht für eine Frühgeburt sprach. Sie wurden bei den adulten Artgenossen belassen, aufgrund deren Nervosität wir aber nicht festzustellen versuchten, welches der beiden $\$$ \& geboren hatte. Am folgenden Tag wurden alle gesammelten Tenrecinen, nach Arten gesondert, in einem Plastikcontainer verstaut und als Begleitgepäck mit Air Madagascar via Paris nach Wien aufgegeben. Aufgrund eines Streiks blieb der Transportkontainer in Paris bei kaltem und feuchtem Dezemberwetter auf offenem Flugfeld stehen und kam erst 3 Tage später nach Wien. Wir mußten befürchten, daß diese empfindliche Tropentiere verendet wären. Dies war nicht der Fall: Sämtliche Tenrecinen (insgesamt 42) hatten überlebt, nur die beiden neonaten $T$. ecaudatus waren verschwunden, d.h. von den adulten gefressen worden. Da diese indessen gegeneinander keinerlei Aggressivität zeigten und wir von Andriatsarafara über ihre, für Insectivora ungewöhnliche Sozialtoleranz unterrichtet waren, wurden sie in unserer Insektivorenstation in geräumigen, miteinander verbundenen und abwechslungsreich eingerichteten Behältern untergebracht.

Am 12. Dezember wurden wieder 2 Jungtiere geboren. Wir mußten annehmen, daß sie von dem anderen $\&$ stammten. Um das Aufkommen dieser für uns kostbaren Tierchen nicht zu gefährden, wurde die nunmehr fünfköpfige Tenrec-Gruppe 
weiterhin in Ruhe gelassen, da uns zufolge langjähriger Insectivorenzucht längst bekannt war, daß besonders primipare Mütter ihre Neonaten nach deren Berührung durch Menschen häufig nicht mehr annehmen. Die ebenso große Gefahr einer händischen Untersuchung der Milchzitzen nach sezernierender Milch wurde deshalb als sinnlos angesehen, weil wir aus den Erfahrungen mit gebärenden Hemicentetes $-\uparrow ९$ wußten, daß die Zitzen wenigstens in den ersten Stunden post partum nicht größer sind als jene nicht trächtiger $\%$ \&. Auch die reptilienhaft wirkende Pseudokloake weiblicher Tenrecinen zeigt unmittelbar post partum keinerlei Tumeszenz.

In den nächsten Tagen hielten sich die 3 adulten Tenrecs zusammen mit den beiden Jungtieren tagsüber, dicht gedrängt, in einem Schlafkistchen auf $(30 \times 30 \times 10 \mathrm{~cm}$, mit $30 \times 10 \mathrm{~cm}$ Vorraum). Die Kleinen schienen zu gedeihen und liefen nachts sehr bald eifrig umher. Unerwarteterweise wurden am 22. Dezember zehn weitere Jungtiere geboren. Sie lebten mit den drei adulten Exemplaren und den 10 Tage älteren Jungtieren zusammen und entwickelten sich gut (Abb. 3).

Es ist anzunehmen, aber nicht sicher, daß die insgesamt 14 Jungtieren von beiden $\uparrow ९$ am 2 , 12. und 22. Dezember geboren wurden. In diesem Falle müssen beide schon vor dem Fang befruchtet worden sein.

Theoretisch könnten aber alle drei Austreibungen von ein und demselben $\%$ stammen, was indessen das Geschehen physiologisch oder geburtsbiologisch noch rätselhafter erscheinen läßt. Da die Tragzeit nach Louwman und Eisenberg \& Muckenhirn nicht bloß 20 Tage betragen kann, kann auch der dritte Wurf(teil?) (vom 22. Dezember) nicht knapp post partum des ersten (vom 2. Dezember) gezeugt worden sein. Ob dem Gesamtphänomen eine Superfecundation, eine Superfetation, ein Blastocystenreservoir oder eine Embryostase zugrundeliegt, ist unklar.

Von den beiden am 10. Dezember geborenen Jungtieren magerte das kleinere aus unbekannter Ursache ab, trank aber von einem $९$ (das nicht notwendigerweise seine Mutter gewesen sein muß) und leckte am 31. Dezember erstmalig am Futter der adulten Tiere. Es starb am 6. Januar 1986 (Abb. 4). Sein Geschwister blieb trotz seiner relativen Größe in der Gruppe $\left(1 \circ^{\circ}, 2\right.$ ९ , 1 größeres und

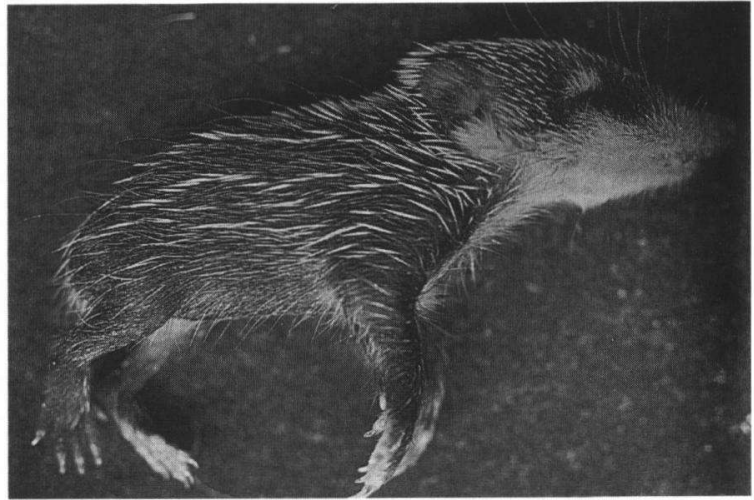

$A b b$. 4. Im Wachstum etwas zurückgebliebenes Jungtier von Tenrec ecaudatus. KRL etwa $12 \mathrm{~cm}, 25$ Tage alt. Beachte das Größenverhältnis der Kopf- zur Körperlänge, die hellen Längsstreifen und die dazwischenstehenden Haare. (Photographie W. Poduschka.)

10 kleinere Jungtiere) integriert und kam problemlos auf.

Unterschiedliche Reifegrade der Einzelfeten bei Mehrlingsgeburten sind von verschiedenen, zumeist allerdings von domestizierten Säugetieren seit langem bekannt. Für die Unterfamilie Tenrecinae gibt es diesbezüglich eine Erwähnung von Gould \& Eisenberg (1966), sowie eine speziell darauf bezogene Arbeit bei Setifer setosus von Poduschka \& Poduschka (1982). In Würfen von Hemicentetes und Echinops kommt zwar gelegentlich ein kümmerndes Jungtier vor, doch sind die Entwicklungsunterschiede zwischen Wurfgeschwistern in der Regel geringfügig und werden zumeist in wenigen Tagen ausgeglichen.

Von den bei mir im Dezember 1985 geborenen T. ecaudatus wurden $2 O^{\circ} O^{\circ}$ und $1 \odot$ an den Zoo Wilhelma/Stuttgart (Dir. Prof. Neugebauer) abgegeben und brachten dort am 30. September 1986 einen Wurf von 28 lebenden und einem toten Jungtier (Neugebauer, mündl. Mitt.). Da das Muttertier somit nur 9 Monate alt war, wird entgegen meiner oben erwähnten Vermutung bezeugt, daß auch Erstwürfe nicht notwendigerweise klein sein müssen.

\section{Diskussion}

Die Tatsache, daß sich nur bei zweien der vier Tenrecinen-Gattungen die referierten fortpflan- 


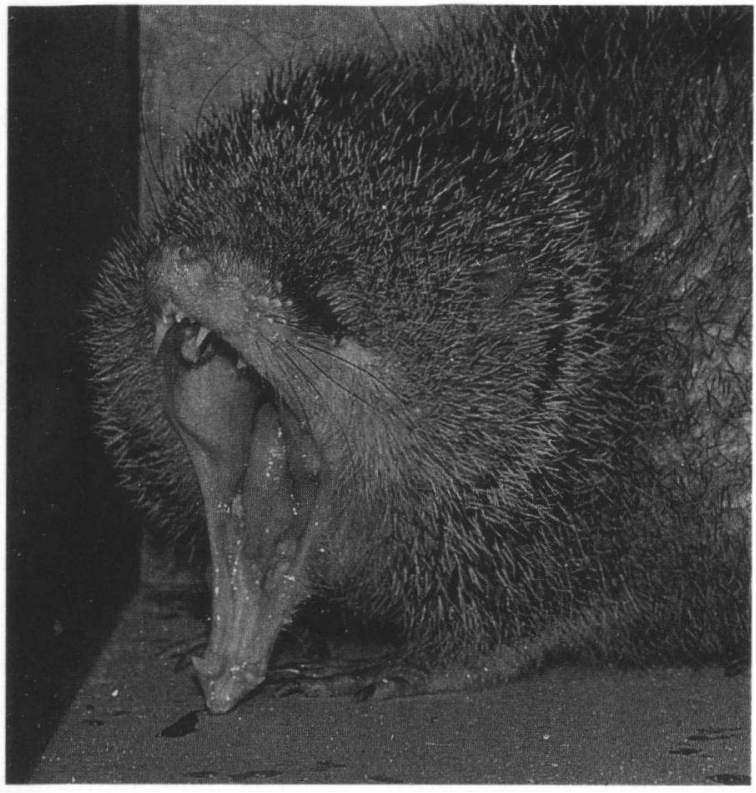

Abb. 5. Tenrec ecaudatus. Das gereizte $\sigma$ droht durch Offnung der Kiefer bis zu $90^{\circ}$ und gloriolenähnliches Sträuben der Nackenstacheln. Beachte die mächtigen Kaumuskeln an den Medianseiten der Wangen. (Photographie W. Poduschka.)

zungsphysiologischen Besonderheiten finden, sagt noch nichts aus über den phylogenetischen Verwandtschaftsgrad. Heim de Balsac (1972) wirft die Frage auf, ob man in der hohen Follikelzahl bei Tenrec und Hemicentetes einen Atavismus aus der Reptilienzeit (Therapsiden) sehen kann. Sie könnte vermutlich besser beantwortet werden, gäbe es mehr Wissen über die Biologie der anderen Unterfamilie (Oryzorictinae). Ich fand nämlich Anzeichen dafür, daß auch bei ihnen im Vergleich zur Körpergröße der Muttertiere bemerkenswert große Würfe vorkommen können (Poduschka, unveröff.).

Bezüglich der hier geschilderten, miteinander korrelierbaren Eigenheiten bei Tenrec und Hemicentetes darf vorläufig nur vermutet werden, daß sie eine auf Wechselwirkungen von Selektionsdruck und Adaption zurückzuführende Sekundärentwicklung sind. So könnte man die in der Klasse Säugetiere einmalige Wurfstärke bei $T$. ecaudatus als Paradebeispiel einer ausgeprägte $r$-Strategie ansehen: Aufgrund seiner Größe wird $T$. ecaudatus nicht nur von Menschen, sondern - wenigstens die Jungtiere - auch von den größeren Schleichkatzen
Madagascars gejagt, die Wurfzahl gleicht aber die zu erwartenden Verluste durch Freßfeinde aus.

Es soll noch darauf hingewiesen werden, daß in dieser Arbeit nur 14 Tenrec-Würfe aufgezählt werden konnten, von denen 2 (bzw. 3?) in Intervallen zur Welt kamen. Das sind immerhin mindestens $14.28 \%$. Es fragt sich, ob solche Partial-Austreibungen bei Tenrec (und auch bei Hemicentetes ?) vielleicht häufiger vorkommen, jedoch bei der Seltenheit ihrer Zucht unter Haltungsbedingungen bisher der Aufmerksamkeit entgingen. $\mathrm{Da}$ sie in freier Wildbahn nicht beobachtet werden können, wurde schon erwähnt.

\section{Zur Längsstreifung von Hemicentetes}

Das merkwürdige Zusammentreffen, daß nur juvenile Tenrec ecaudatus wie die etwa gleichgroßen adulten Hemicentetes und seine Jungen längsgestreift sind, wurde von Eisenberg \& Gould (1970) als Neotenie bei Hemicentetes vermutet, hingegen weist Vogel (1996, briefl. Mitt.) zurecht darauf hin, daß dies besser als Progenesis, eine andere Form der Pädomorphosis im Sinne von Futuyma (1986), gedeutet werden sollte. In der Auswirkung könnte es als Mimikry zugunsten von Hemicentetes angesehen werden.

Der Sinn dieser Mimikry mag darin liegen, daß im Gegensatz zu Tenrec - adulte Hemicentetes aufgrund ihrer geringen Größe trotz ihrer detachierbaren Stacheln mit Widerhaken für größere Feinde leichte Beute sind. Sie können aber von ihrer Streifung insofern profitieren, daß sie von Freßfeinden für juvenile Tenrec gehalten werden. Da sich diese von ihren Elterntieren, die sie durch mächtige Kaumuskeln und das reptilienhaft weite Aufreißen der Kiefer effizient verteidigen (Abb. 5), kaum entfernen und dicht aneinandergedrängt mit ihnen fouragieren, sind wohl auch juvenile Hemicentetes trotz, bzw. gerade wegen ihrer auffälligen Zeichnung, einigermaßen geschützt. Dies umso mehr, als auch junge Hemicentetes eine dicht aneinandergedrängte Lokomotion bevorzugen (Eisenberg, 1964; Eisenberg \& Gould, 1970). Dazu kommt noch die - allerdings bei den Neonaten aller vier Tenrecinengattungen feststellbare - Fähigkeit von Nestflüchtern, spätestens am 2. Lebenstag mobil zu sein. 


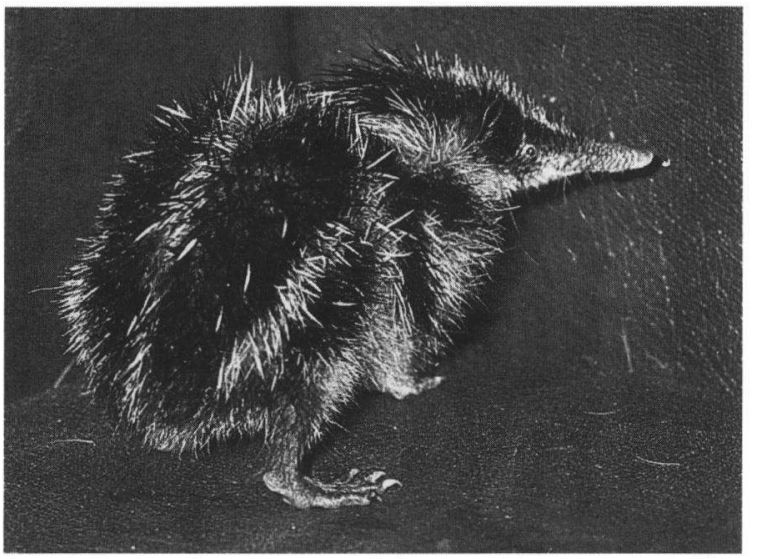

Abb. 6. Hemicentetes semispinosus. Beachte die gegenüber Abb. 7 schmäleren, hellen Längsstreifen und den durch die auf diesem Bild nicht gesträubten Nackenstacheln länger und schmäler wirkenden Kopf. (Photographie W. Poduschka.)

$\mathrm{Zu}$ erwähnen ist, daß H. semispinosus (Abb. 6) durch seine schmäleren Längsstreifen den juvenilen Tenrec ecaudatus wesentlich ähnlicher ist als $H$. nigriceps (Abb. 7). Ob dies aussagt, daß die Streifenbildung des erstgenannten Taxons die ursprüngliche ist und die des zweiten eine (jüngere) Hypertrophie davon, wage ich nicht zu entscheiden, zumal die Konsistenz der borstenartigen Körperbedeckungen bei beiden Arten sehr unterschiedlich ist.

\section{Danksagung}

Großen Dank schulde ich der Stiftung Volkswagenwerk, die meine Arbeiten großzügig unterstützte. Ebenso danke ich Herrn Prof. emer. Dr. Dr. h.c. Dietrich Stark (Frankfurt a.M.) für Ratschläge und praktische Hilfe. Prof. Dr. Kurt Arbeiter (Tierärztliche Universität Wien) und Prof. Dr. Wolfgang Schleidt (University of Maryland und Universität Wien) danke ich sehr herzlich für die kritische Durchsicht des Manuskripts. Ganz besonders bin ich Herrn Prof. Dr. Peter Vogel (Université de Lausanne) für die bereichernde Kritik aufrichtig dankbar. Dank auch an Prof. Dr. Roland Albignac (Université de Montpellier und Université de Antananarivo) für unschätzbare Ratschläge in Madagascar. Dem exquisiten Kreis von Fachkollegen, die zu praktischer Hilfe stets bereit waren, möchte ich hier ebenso danken: Dr. J.F. Eisenberg (Universität und Museum Gainesville/Florida), Dr. E. Gould (Zoo Washington), Dr. I. Eibl-Eibesfeldt (Max Planck-Institut Erling-Andechs), Prof. Dr. Jean-Jacques Petter (Directeur, Laboratoire de Recherche sur les Prosimiens, Brunoy/Frankreich, u.a.m.

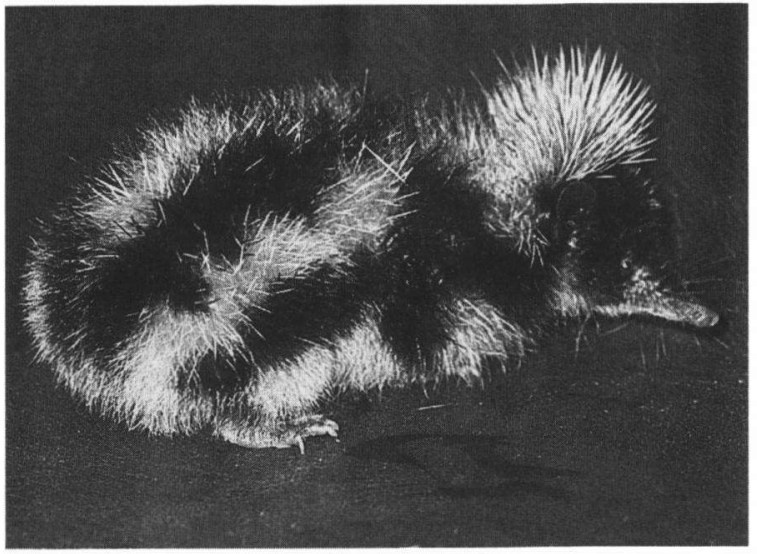

Abb. 7. Hemicentetes nigriceps. Sowohl die Nackenstacheln als das im Vergleich zu Abb. 6 wesentlich üppigere Borstenkleid sind gesträubt und geben dem Tier ein kompakteres Aussehen. (Photographie W. Poduschka.)

\section{Literatur}

Andriatsarafara, F.R., 1981. Quelques observations sur l'ontogénie et le comportement de Tenrec ecaudatus (Schreber 1777) en captivité; comparaisons avec les données connues chez d'autres insectivores: 1-22; 1-23 (Université de Madagascar).

Bauchot, R. \& H. Stephan, 1964. Le poids encéphalique chez les Insectivores malgaches. Acta zool., 45: 63-75.

Bauer, K., 1960. Die Säugetiere des Neusiedlersee-Gebietes (Österreich). Bonner zool. Beitr., 11(2-4): 141-344.

Bluntschli, H., 1932. In den Urwäldern auf Madagascar. III. Im Küstenwald des mittleren Ostens. Reise in das Bergwaldgebiet. Die Umschau, 36: 891.

Bluntschli, H., 1937. Die Frühentwicklung eines Centetinen (Hemicentetes semispinosus Cuv.). Revue suisse Zool., 44: 271-282.

Bluntschli, H., 1938a. Le développement primaire et la formation d'un placenta perforé très compliqué et du type labyrinthe chez Hemicentetes. Bull. Acad. malgache, 20: 73-75.

Bluntschli, H., 1938b. Le développement primaire et l'implantation chez un Centétiné (Hemicentetes). C.r. Assoc. Anat. Paris, $33^{\text {me }}$ réunion: 1-8 (Bâle).

Breslau, E., 1910. Der Mammarapparat (Entwicklung und Stammesgeschichte). Ergebn. Anat. Entwicklungsgesch., Wiesbaden, 19: 275-349.

Buffon [G.-L. Leclerc de], 1764. Histoire naturelle, générale et particulière, avec la description du cabinet du roi, 12: 438-441, pls. LVI-LVII (Imprimerie Royale, Paris).

Dobson, G.E., 1882. A monograph of the Insectivora, systematic and anatomical. Part I-III. Erinaceidae, Centetidae, and Solenodontidae: i-iv, 1-172, pls. 1-28 (John van Voorst, London).

Eibl-Eibesfeldt, I., 1965. Das Duftmarkieren des Igeltanrek (Echinops telfairi Martin). Z. Tierpsychol., 22: 810-812. 
Eisenberg, J.F., 1964. Experimental techniques in the analysis of grouping tendencies in rodents and insectivores. Amer. Zoologist, 4(3)August: 13.

Eisenberg, J.F. \& E. Gould, 1967. The maintenance of tenrecoid insectivores in captivity. Int. Zoo Yearb., 7: 194196.

Eisenberg, J.F. \& E. Gould, 1970. The tenrecs: A study in mammalian behavior and evolution. Smithson. Contr. Zool., 27: i-vi, 1-138.

Eisenberg, J.F. \& N. Muckenhirn, 1968. The reproduction and rearing of tenrecoid insectivores in captivity. Int. Zoo Yearb., 8: 106-110.

Firbas, W. \& W. Poduschka, 1971. Beitrag zur Kenntnis der Zitzen des Igels (Erinaceus europaeus Linné). Säugetierkundl. Mitt., 19: 39-44.

Francke, H., 1961. Gefangenschaftsbeobachtungen an Hemicentetes semispinosus. Sitz. Ber. Ges. Naturforsch. Fr. Berlin, (N.F.) 1: 118-123.

Futuyma, D.J., 1986. Evolutionary biology (2nd ed.): i-xii, 1-600 (Sinauer Associates, Sunderland Mass.)

Geoffroy Saint-Hilaire, I., 1839. Tanrec. Cuv. Centetes, Illig. et Ericule. Ericulus. Magasin Zool. Paris, ${ }^{2} 1839$; Mammifères, pls. $1-4$.

Goetz, R.H., 1936. Studien zur Placentation der Centetiden. I. Eine Neu-Untersuchung der Centetes Placenta. Z. Anat. Entwicklungsgesch., 106: 325-342.

Gould, E. \& J.F. Eisenberg, 1966. Notes on the biology of the Tenrecidae. J. Mammal., 47(4): 660-685.

Grandidier, G. \& G. Petit, 1932. Zoologie de Madagascar: [iiii], 1-258, pls. I-XLVIII (Soc. d'Editions geogr., marit. et coloniales, Paris).

Heim de Balsac, H., 1972. Insectivores. In: R. Battistini \& H. Heim de Balsac (eds.), Biogeography and ecology in Madagascar. Monographiae biol., 21: 629-660 (W. Junk, The Hague).

Honegger, R.E. \& W. Noth, 1966. Beobachtungen bei der Aufzucht von Igeltanreks Echinops telfairi Martin. Zool. Beitr. (N.F.) 12(2): 191-218.

Jentinck, F.A., 1879. On the hedgehogs from Madagascar. Notes Leyden Mus., 1: 137-151.

Lacépède [B.G.E. de la Ville], 1799. Tableau des divisions, sous-divisions, ordres et genres des Mammifères: 1-18 (Plassan, Paris).

Leche, E., 1907. Zur Entwicklungsgeschichte des Zahnsystems der Säugetiere. II: Phylogenie. Heft 37. Die Familie der Centetidae, Solenodontidae und Chrysochloridae. Zoologica, Stuttgart, 49: 11-96.

Leimgruber, C., 1939. Le crâne primordial d'un embryon de Centetes ecaudatus. Biomorphosis, 1: 545-585.

Louwman, J.W.W., 1973a. Breeding the Tailless Tenrec Tenrec ecaudatus at Wassenaar Zoo. Int. Zoo Yearb., 13: 125-126.

Louwman, J.W.W., 1973b. Onze reuzentanrek fokkerij. Parknieuws, Dierenpark Wassenaar, 4: 1-5.

Mallinson, J.J.C., 1968. Conservation of Madagascan fauna in captivity - the Tenrec collection. Annual Rep. Jersey Wildl. Preserv. Trust, 5: 51-53.

Mallinson, J.J.C., 1972. Establishing mammal gestation peri- ods at the Jersey zoological park. Annual Rep. Jersey Wildl. Preserv. Trust, 9: 62-65.

Mivart, St.G., 1871. On Hemicentetes, a new genus of Insectivora, with some additional remarks on the osteology of that order. Proc. zool. Soc. Lond., 1871: 58-71.

Petter, J.-J. \& A. Petter-Rousseaux, 1963. Notes biologiques sur les Centetinae. Terre Vie, 1: 66-80.

Poduschka, W., 1974. Fortpflanzungseigenheiten und Jungenaufzucht des Grossen Igel-Tenrek Setifer setosus (Froriep 1806). Zool. Anz., 193: 145-180.

Poduschka, W. \& C. Poduschka, 1982. Über eine Frühgeburt bei Setifer setosus (Froriep 1806) (Insectivora: Tenrecinae). Säugetierkundl. Mitt., 30: 63-68.

Racey, P.A. \& M.E. Nicoll, 1984. Mammals of the Seychelles. In: D.R. Stoddart (ed.), Biogeography and ecology of the Seychelles Islands. Monographiae biol., 55: $607-626$ (W. Junk, The Hague, Boston, Lancaster).

Rand, A.L., 1935. On the habits of some Madagascar mammals. J. Mamm., 16(2): 89-104.

Riordan, D.V., 1972. Reproduction in the Spiny Hedgehog Tenrec Setifer setosus and the Pigmy Hedgehog Tenrec Echinops telfairi. Annual Rep. Jersey Wildl. Preserv. Trust, 9: 18-25.

Romer, A.S. \& T.S. Parsons, 1983. Vergleichende Anatomie der Wirbeltiere. 5., neubearb. Aufl.: i-xv, 1-624 (Paul Parey, Hamburg u. Berlin).

Starck, D., 1974. Die Säugetiere Madagascars, ihre Lebensräume und ihre Geschichte. Sitzungsber. wiss. Ges. Johann Wolfgang Goethe Univ. Frankfurt am Main, 11(3): 1-124 (Steiner, Wiesbaden).

Starck, D., 1982. Vergleichende Anatomie der Wirbeltiere auf evolutionsbiologischer Grundlage. III. Organe des aktiven Bewegungsapparates, der Koordination, der Umweltbeziehung, des Stoffwechsels und der Fortpflanzung: i-xx, 1-1107 (Springer Verlag, Berlin/Heidelberg/New York).

Strauss, F., 1939a. Die Befruchtung und der Vorgang der Ovulation bei Ericulus aus der Familie der Centetiden. Biomorphosis, 1: 281-312.

Strauss, F., 1939b. Die Bildung des Corpus luteum bei Centetiden. Biomorphosis, 1: 489-543.

Strauss, F., 1942. Vergleichende Beurteilung der Placentation bei den Insectivoren. Revue suisse Zool., 50: 269282.

Strauss, F., 1943. Die Placentation von Ericulus setosus. Revue suisse Zool., 50(2): 17-87.

Strauss, F., 1944. Die Bedeutung des placentalen Blutbeutels in vergleichender Betrachtung. Revue suisse Zool., 51 (14): 360-368.

Strauss, F., 1946. Ein Deutungsversuch des uterinen Zyklus von Ericulus. Revue suisse Zool., 53: 511-517.

Weber, M. [W.C.], 1927-1928. Die Säugetiere. Einführung in die Anatomie und Systematik der recenten und fossilen Mammalia, 2. Auflage. I (1927), Anatomischer Teil unter Mitwirkung von H.M. de Burlet: i-xv, 1-444; II (1928), Systematischer Teil unter Mitwirkung von O. Abel: ixxiv, 1-898 (Gustav Fischer, Jena).

Eingegangen am 14. Februar 1996 